\title{
Motion-Induced Perceptual Extrapolation of Blurred Visual Targets
}

\author{
Yu-Xi Fu, Yaosong Shen, and Yang Dan \\ Division of Neurobiology, Department of Molecular and Cell Biology, University of California, Berkeley, California 94720
}

In the motion-extrapolation hypothesis, the visual system can extrapolate the instantaneous position of a moving object from its past trajectory. The existence of such a mechanism in human vision has been intensely debated. Here, we show compelling perceptual extrapolation of both first- and secondorder moving stimuli, the magnitude of which depends on blurring of the visual target. The spatiotemporal characteristics of the extrapolation can be quantitatively accounted for by a

Processing delay in the neural pathway on the order of tens of milliseconds should cause a significant offset between the perceived and the actual positions of a moving object. An appealing hypothesis is that the visual system can compensate for the neural delay and reduce the perceptual misalignment by extrapolating the trajectory of the moving object (Nijhawan, 1994, 1997). Evidence for motion extrapolation originally came from the flash-lag illusion in which the position of a moving object is perceived to be ahead of a briefly flashed object when they are physically colocalized at the time of the flash (MacKay, 1958; Nijhawan, 1994, 1997). However, recent studies of this illusion have yielded results that are inconsistent with the motion-extrapolation model (Baldo and Klein, 1995; Purushothaman et al., 1998; Lappe and Krekelberg, 1998; Whitney and Murakami, 1998; Krekelberg and Lappe, 1999, 2000; Brenner and Smeets, 2000; Eagleman and Sejnowski, 2000; Whitney et al., 2000). In particular, Eagleman and Sejnowski (2000) found no perceived displacement between the flashed and the moving targets if the latter stopped at the time of the flash (also see Krekelberg and Lappe, 2000), directly contradicting the prediction of the motion-extrapolation model. In this study, we have used a similar motion-stop paradigm but different types of visual targets to reexamine the existence of motion-induced perceptual extrapolation. We found compelling extrapolation of moving targets with blurred edges, and this effect was general for both first- and secondorder motion stimuli. The dependence of the motion-induced perceptual extrapolation on the blur and the velocity of the target distinguishes it from the motion-extrapolation mechanism originally proposed to explain the flash-lag illusion (Nijhawan, 1994). These spatiotemporal characteristics, however, can be quantitatively accounted for by a simple model based on the temporally biphasic neuronal response, a mechanism that

\footnotetext{
Received June 1, 2001; revised July 23, 2001; accepted July 24, 2001.

This work was supported by grants from the National Science Foundation and the National Eye Institute. We thank Dr. Gerald Westheimer for helpful discussions.

Correspondence should be addressed to Dr. Yang Dan, Division of Neurobiology, Department of Molecular and Cell Biology, University of California, Berkeley, California 94720. E-mail: ydan@uclink4.berkeley.edu

Copyright (ㄷ) 2001 Society for Neuroscience $\quad 0270-6474 / 01 / 210001-05 \$ 15.00 / 0$
}

simple model based on temporally biphasic neuronal response, a property widely observed among sensory neurons. Thus, motion-induced perceptual extrapolation exists in human vision, and spatial blurring is an important factor in the interaction between motion and perceptual localization.

Key words: psychophysics; motion extrapolation; secondorder motion; edge; blur; localization; biphasic

has been used to account for motion extrapolation (Berry et al., 1999).

After this work had been completed, it came to our attention that Whitaker et al. (1998) have reported in abstract form the perceptual extrapolation of luminance-defined moving Gaussian target that depends on the Gaussian width.

\section{MATERIALS AND METHODS}

Psychophysical experiments. Visual stimuli were generated with a personal computer with a Leadtek Winfast 3D L3100 graphics board and presented at a refresh rate of $120 \mathrm{~Hz}$. Viewing was binocular from $72 \mathrm{~cm}$. Each of the two horizontal stripes (see Figs. $1 A-C, 4 A, C$ ) was $24 \times 1.6^{\circ}$. The fixation square $\left(0.32 \times 0.32^{\circ} ; 90 \mathrm{~cd} / \mathrm{m}^{2}\right)$ between them was present throughout each session, in which a staircase procedure (Gescheider, 1997) was used to measure the perceptual displacement. Although the two targets always moved in opposite directions, each target moved leftward and rightward in equal numbers of sessions (randomly mixed) to avoid bias, and data from all these sessions were combined. The mean and SE of the perceptual extrapolation under each stimulus condition were obtained from the end points measured in at least 10 sessions. The $100 \mathrm{msec}$ interval between the stop and the disappearance of the targets (see Results) made it easier for the subjects to perceive the end positions of the targets, but eliminating this period did not significantly affect the magnitude of the perceptual extrapolation $(t$ test, $p>0.60$ for subjects $\mathrm{YF}$ and $\mathrm{HG}$, measured at a velocity of $4 \% \mathrm{sec}$; target luminance, $90 \mathrm{~cd} / \mathrm{m}^{2}$; background, $10 \mathrm{~cd} / \mathrm{m}^{2}$; and Gaussian width, $4^{\circ}$ ).

Population neuronal response model. The model consisted of an array of 500 neurons in a visuotopically organized circuit whose receptive fields tile the visual field along the dimension of target motion. The response of the $i$ th neuron, $r_{i}(t)$, is modeled as a linear convolution of the stimulus $s(x, t)$ and the spatiotemporal receptive field $k_{i}(x, t)$ (modeled as retinal

This article is published in The Journal of Neuroscience, Rapid Communications Section, which publishes brief, peerreviewed papers online, not in print. Rapid Communications are posted online approximately one month earlier than they would appear if printed. They are listed in the Table of Contents of the next open issue of JNeurosci. Cite this article as: JNeurosci, 2001, 21:RC172 (1-5). The publication date is the date of posting online at www.jneurosci.org.

http://www.jneurosci.org/cgi/content/full/5710 
ganglion cells with concentric center-surround receptive fields), followed by a rectification at threshold $\theta$ :

$$
\begin{aligned}
r_{i}(t) & =\max \left[\int_{-\infty}^{\infty} d x \int_{-\infty}^{t} d t^{\prime} s\left(x, t^{\prime}\right) k_{i}\left(x, t-t^{\prime}\right)-\theta, 0\right] \\
k_{i}(x, t) & =\left[e^{-\left(x-x_{i}\right)^{2} / 2 \sigma_{k 1^{2}}}-A e^{-\left(x-x_{i}\right)^{2} / 2 \sigma_{k 2^{2}}}\right] \times\left[t e^{-a t}-c^{2} t e^{-b t}\right],
\end{aligned}
$$

where $A$ is the strength of the receptive field surround relative to the center, $\sigma_{k 1}$ and $\sigma_{k 2}$ determine the widths of the receptive field center and surround, respectively, $x_{i}$ is the position of the receptive field center of the $i$ th neuron, $a$ determines the time constant of the initial excitatory component, and $b$ and $c$ determine the time constant and the relative strength of the delayed inhibitory component of the impulse response, respectively. The stimuli with different types of moving targets are described in Figure 1. The population response profile (see Fig. $3 B-D$, middle panel) was predicted by plotting the responses of the array of neurons $\left\{r_{i}\right\}$ versus their receptive field positions $\left\{x_{i}\right\}$. The values of $A$ $(0.2), \sigma_{k 1}\left(0.1^{\circ}\right), \sigma_{k 2}\left(0.2^{\circ}\right)$, and $a\left(0.0625 \mathrm{msec}^{-1}\right)$ were roughly estimated on the basis of values in the literature and physiological data from our own laboratory. We left $b, c$, and $\theta$ (spike threshold) as free parameters whose values were adjusted to fit the data. The question of which aspect of the population response profile is used for perceptual judgment of the position of a blurred target is not yet resolved (Watt and Morgan, 1983). In Figure $3 F$, we used the center of mass of the simulated response profile at the time of target disappearance ( $100 \mathrm{msec}$ after stop) to represent perceived position. When we used other measures such as the zerocrossing positions of the second derivative of the response profile as perceived position, the model could also fit the data, although with different sets of parameters. Note that the response property of each neuron in this model is linear (except for a rectification), and it does not include the contrast-gain control mechanism used in Berry et al. (1999). In preliminary studies, we found that the gain-control mechanism was not necessary to account for the target-width and velocity dependence of the perceptual extrapolation we have observed, nor was it sufficient when implemented in the absence of the biphasic response property. However, when implemented together with the biphasic response property, it may help to explain the dependence of the effect on target luminance (see Fig. 2B).

\section{RESULTS}

\section{Perceptual extrapolation of blurred moving targets}

The stimuli consisted of a pair of visual targets moving horizontally at the same speed but in opposite directions. After a few seconds, both targets stopped for $100 \mathrm{msec}$ and then disappeared. For a pair of Gaussian targets that stopped in perfect alignment with each other, subjects perceived a conspicuous misalignment between their stopping positions in the direction of motion (Fig. $1 A$ ). The magnitudes of the misalignment measured for four subjects (two naïve subjects and two authors) were $1.30 \pm 0.09^{\circ}$ $(\mathrm{HG}), 1.56 \pm 0.14^{\circ}(\mathrm{KB}), 1.44 \pm 0.06^{\circ}(\mathrm{YF})$, and $0.61 \pm 0.05^{\circ}$ (YD), respectively. Significant misalignment in the same direction was also observed for all subjects ( $p<0.05, t$ test) when they compared the stopping position of a moving target with the position of a flashed target, a paradigm used in previous studies to measure motion extrapolation (Eagleman and Sejnowski, 2000; Krekelberg and Lappe, 2000). Because in these motion-stop experiments, the moving targets were never physically presented beyond their stopping positions along the path of motion, the observed perceptual displacement cannot be accounted for by the differential latency (Purushothaman et al., 1998; Whitney and Murakami, 1998; Whitney et al., 2000), the temporal integration (Lappe and Krekelberg, 1998; Krekelberg and Lappe, 1999, 2000), or the postdiction (Eagleman and Sejnowski, 2000) models used to explain the flash-lag illusion. Instead, it represents spatial extrapolation of the target position along the trajectory of motion.

Interestingly, we noticed that the perceptual extrapolation depends on spatial blurring of the visual target. For a pair of targets
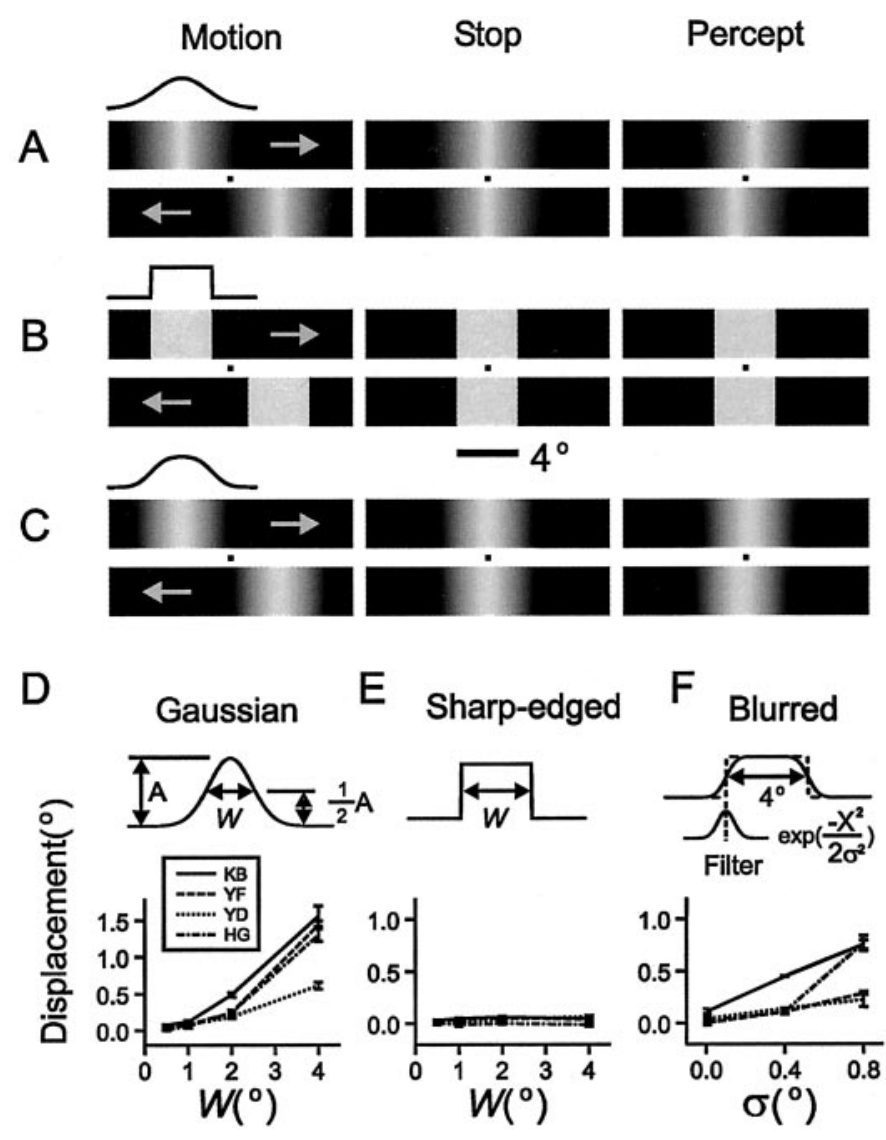

Figure 1. Spatial extrapolation of moving targets with different spatial profiles. $A$, A pair of targets with Gaussian luminance profiles, $L(x, t)=$ $B+A \exp \left[-(x-v t)^{2} / 2 \sigma^{2}\right]$, where $L$ is the luminance, $x$ is position, $t$ is time, $B$ is background $\left(10 \mathrm{~cd} / \mathrm{m}^{2}\right), B+A$ is the peak luminance of the Gaussian target $\left(90 \mathrm{~cd} / \mathrm{m}^{2}\right), v$ is velocity $(4 \% \mathrm{sec})$, and $\sigma$ is $\mathrm{SD}$ of the Gaussian function. The width at half height of the target $(W=2.35 \sigma)$ was $4^{\circ}$. The curve above represents the spatial luminance profile, $L(x)$. The targets moved for $2 \mathrm{sec}(l e f t)$, stopped for $100 \mathrm{msec}$ when they reached vertical alignment (middle), and disappeared. The fixation point (small square between the two targets) was present throughout every session. All subjects that were tested reported a clear perceived misalignment between the two targets in the direction of motion. The percept depicted on the right reflects the average displacement of the four subjects measured with a staircase procedure. $B$, Left and middle, the motion-stop stimulus containing a pair of sharp-edged targets (background, $10 \mathrm{~cd} / \mathrm{m}^{2}$; target luminance, $90 \mathrm{~cd} / \mathrm{m}^{2}$; width, $4^{\circ}$; velocity, $4^{\circ} / \mathrm{sec}$ ). Right, the average perceptual displacement from the four subjects. $C$, The targets in $B$ blurred by convolution with a Gaussian filter $\left(\sigma=0.8^{\circ}\right)$. The percept (right) represents the average displacement of the four subjects. $D$, Perceptual displacement as a function of the width at half height of the Gaussian targets $(W)$. Positive values represent a displacement in the direction of motion. E, Perceptual displacement as a function of the width of the sharp-edged targets. $F$, Displacement as a function of the $\mathrm{SD}, \sigma$, of the Gaussian filter used to blur the sharp-edged targets. Note that $E$ and $F$ are plotted on a different scale from $D$. Error bars indicate SEM.

with sharp edges, the perceptual displacement was near zero (Fig. 1B) $\left(\mathrm{HG},-0.01 \pm 0.01^{\circ} ; \mathrm{KB}, 0.05 \pm 0.01^{\circ}\right.$; YF, $0.04 \pm 0.01^{\circ}$; YD, $\left.0.07 \pm 0.01^{\circ}\right)$. Blurring the edges of these targets, however, significantly increased the displacement (Fig. 1C) (HG, $0.76 \pm$ $0.07^{\circ}$; KB, $0.75 \pm 0.04^{\circ}$; YF, $0.29 \pm 0.01^{\circ}$; YD, $0.22 \pm 0.07^{\circ}$ ). To further characterize the effect of blurring, we measured the displacement as a function of the width of the Gaussian target and found it to increase monotonically between 0.5 and $4^{\circ}$ for all four subjects (Fig. 1D). For sharp-edged targets, the displacement showed little dependence on the target size (Fig. 1E), but a 

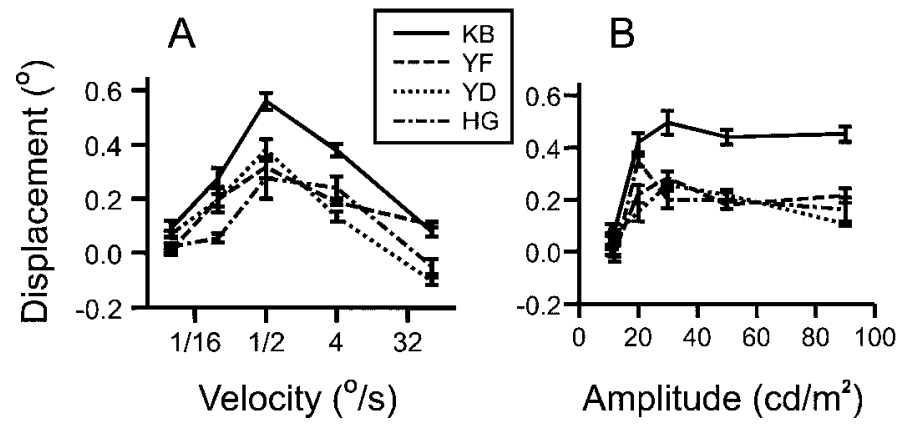

Figure 2. Dependence of the perceptual extrapolation of four subjects on the velocity and the peak luminance of the Gaussian targets. $A$, The perceptual displacement as a function of the target velocity. In these experiments, the targets moved for $4 \mathrm{sec}$ before stopping. $B$, The displacement as a function of the peak luminance of the Gaussian targets at a fixed background of $10 \mathrm{~cd} / \mathrm{m}^{2}$. Peak luminance at 11, 12, 20, 30,50 , and $90 \mathrm{~cd} / \mathrm{m}^{2}$ correspond to peak contrast [Michaelson contrast, defined as $\left.\left(L_{\text {max }}-L_{\text {min }}\right) /\left(L_{\text {max }}+L_{\text {min }}\right)\right]$ at $0.05,0.09,0.33,0,50,0.67$ and 0.80 , respectively. Error bars indicate SEM.

significant dependence on blurring of the target edges (Fig. $1 F$ ). Thus, the magnitude of the perceptual displacement depends strongly on the spatial blurring of the visual targets, which may explain why previous studies using sharply defined objects did not show significant extrapolation (Eagleman and Sejnowski, 2000; Krekelberg and Lappe, 2000).

\section{Dependence of the perceptual extrapolation on target velocity and luminance}

The three parameters that uniquely define a moving Gaussian target, i.e., width, velocity, and peak amplitude, may all affect the magnitude of the perceptual extrapolation. In addition to the dependence on the Gaussian width (Fig. 1D), which is a measure of target blur, we also characterized the displacement as a function of the target velocity and luminance amplitude. Dependence on the target velocity was measured between 0.03 and $64 \% \mathrm{sec}$ at fixed peak luminance of $90 \mathrm{~cd} / \mathrm{m}^{2}$, background of $10 \mathrm{~cd} / \mathrm{m}^{2}$, and target width of $2^{\circ}$. Significant extrapolation was observed over a wide range of velocities with a maximum at $\sim 0.5^{\circ} / \mathrm{sec}$ (Fig. $2 A$ ). Such velocity dependence is different from that of the flash-lag illusion, which increases approximately linearly with the target velocity (Nijhawan, 1994). The dependence on the peak luminance of the Gaussian targets was measured at a fixed background of $10 \mathrm{~cd} / \mathrm{m}^{2}$, target width of $2^{\circ}$, and velocity of $4^{\circ} / \mathrm{sec}$. The effect rose with increasing luminance amplitude and reached saturation at $\sim 20 \mathrm{~cd} / \mathrm{m}^{2}$ (Fig. $2 B$ ). When we switched the luminance polarity of the targets so that they are defined by dark Gaussian profiles on a light background (target peak luminance, $10 \mathrm{~cd} / \mathrm{m}^{2}$; background, $90 \mathrm{~cd} / \mathrm{m}^{2}$; width, $4^{\circ}$; velocity, $4^{\circ} / \mathrm{sec}$ ), significant extrapolation was still observed by all four subjects ( $p<0.0001, t$ test). Thus, the perceptual displacement is determined by the direction of target motion and is independent of the polarity of the luminance gradient.

\section{A neuronal model for the perceptual extrapolation}

What is the mechanism underlying the blur-dependent perceptual extrapolation? In a visuotopically organized neuronal circuit, the spatial profile of the population neural response moves within the circuit when the target moves in the visual field, and the position of the response profile may determine the perceived position of the visual target. In the rabbit and salamander retina, the transient response property and the contrast-gain control mechanism have been shown to shift the response profile of the retinal ganglion cells in the direction of target motion, which has been proposed as a mechanism for motion extrapolation (Berry et al., 1999). To test whether these retinal response properties can account for the blur-dependent perceptual extrapolation observed in this study, we simulated the response of each neuron to the motion-stop stimuli using these response properties and predicted the spatial profile of the population response of an array of neurons (see Materials and Methods). Surprisingly, we found that a simple model incorporating only the biphasic temporal response property (Fig. $3 A$ ) is sufficient to account for the spatiotemporal characteristics of the observed perceptual extrapolation.

Figure 3, $B$ and $C$, shows the simulated population responses of an array of retinal neurons to a moving Gaussian target with monophasic and biphasic temporal response functions (Fig. 3A), respectively. Comparison of the two response profiles shows that the delayed inhibitory component in the biphasic temporal response function caused a shift of the population response profile toward the direction of motion, which could account for the observed perceptual extrapolation (Fig. 3C, bottom panel). For a sharp-edged target, the biphasic response property also distorted the response profile, but caused little shift in its overall position (Fig. 3D). Thus, this model can explain the blur-dependence of the perceptual extrapolation. To further test the spatiotemporal properties of the model, we measured the perceptual extrapolation as a function of both the Gaussian width and target velocity, averaged from data of all four subjects (Fig. $3 E$ ). By adjusting only three parameters (see Materials and Methods), the model well accounted for the data over the entire range of target widths and velocities investigated (Fig. $3 F$ ). Interestingly, a consistent feature of the data among all subjects is that the optimal velocity for perceptual extrapolation increases with the Gaussian width (Fig. $3 E$, white line). This velocity-width inseparability was also a robust feature of the model (Fig. $3 F$ ) and was insensitive to the exact parameters used (data not shown). Taken together, the simple model incorporating the biphasic temporal response property of the retinal neurons is sufficient to account for the observed target-width and velocity dependence of the perceptual extrapolation. Although the luminance-dependence of the extrapolation (Fig. 2B) cannot be accounted for by the linear model, it can be explained if the relative weight of the inhibitory component of the biphasic response increases with the stimulus contrast. Such a property is known to exist in the retina as part of contrast-gain control (Shapley and Victor, 1978), which has been used in a model predicting the response profiles of retinal ganglion cells to moving stimuli (Berry et al., 1999). Similar contrast-dependent enhancement of inhibition may also occur in the visual cortex, because the inhibitory cortical neurons appear to have higher response gains than the excitatory cortical neurons (Somers et al., 1998).

\section{Perceptual extrapolation of second-order moving targets}

Can motion-induced perceptual extrapolation be supported by cortical mechanisms? In addition to the luminance-defined firstorder stimuli as those used above, the visual system can also process contrast- or texture-defined second-order targets $(\mathrm{Ca}$ vanagh and Mather, 1989), which is likely to involve the visual cortex. To test the generality of the motion-induced perceptual extrapolation and whether it can be supported by cortical mechanisms, we repeated the motion-stop experiments using targets defined by contrast or orientation. The contrast-defined stimulus 
A Model

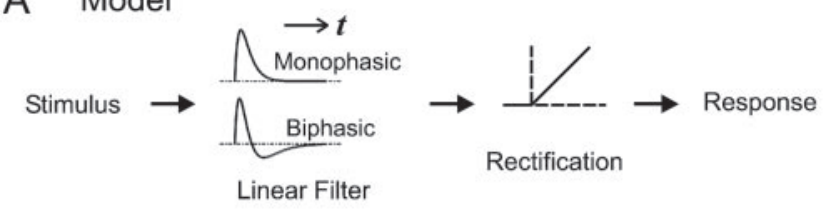

B Monophasic

\section{Biphasic}

\section{Biphasic}
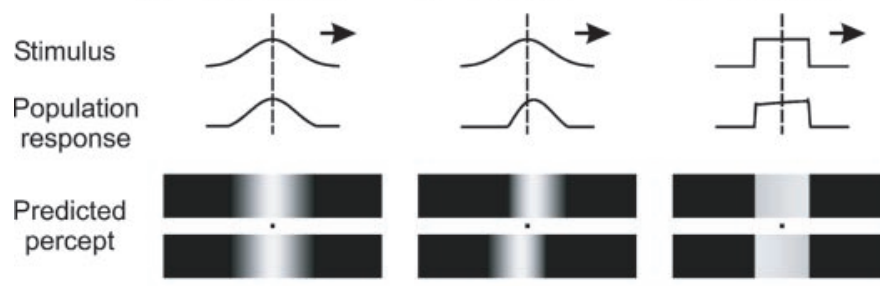

percept

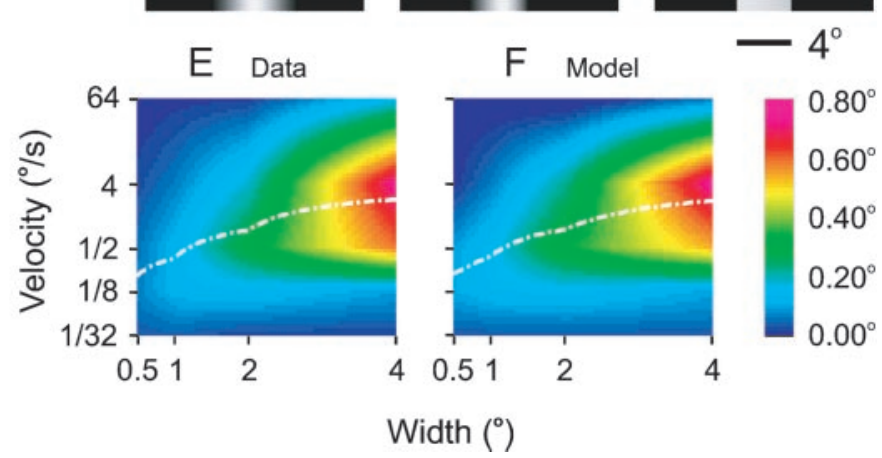

Figure 3. A model of the perceptual extrapolation based on the biphasic temporal responses of visual neurons. $A$, The linear model used to predict the response of each retinal neuron to the stimulus. The stimulus was filtered by the spatiotemporal receptive field of the neuron and then rectified to yield the response. The linear filter shown here represents the temporal impulse response function of the neuron, which is the firing rate of the neuron in response to a brief flash of the stimulus. Illustrated are two types of impulse response functions: monophasic and biphasic. The spatial receptive fields used in the model are omitted here for clarity. $B$, Spatial luminance profile of a Gaussian target (top trace, dashed line indicates peak position), the spatial profile of simulated population neuronal response with a monophasic temporal response function $(c=0, \theta=$ 0.016 , bottom trace), and the predicted percept of the motion-stop stimuli used in our experiments when the pair of targets moving in opposite directions stopped in perfect alignment (bottom panel). In this simple model, the luminance profile of the predicted percept is proportional to the predicted population response profile (trace above). $C$, Luminance profile of the same target shown in $B$ (top trace), the simulated population neuronal response with a biphasic temporal response function (bottom trace), and the predicted perceptual misalignment between the stopping positions of the two targets (bottom panel). Model parameters $(b=0.0025$ $\mathrm{msec}^{-1}, c=0.036, \theta=0.016$ ) were adjusted to fit the data in Figure $1 \mathrm{~A}$. Note that the fitted value for $b$ corresponds to a time constant of $400 \mathrm{msec}$, which is much longer than that known for the retinal responses. It is possible that cortical mechanisms, which are known to have much longer time constants, are involved in the observed perceptual shift. Alternatively, this long time constant may reflect a slow retinal process such as light or contrast adaptation (Berry et al., 1999). D, Spatial luminance profiles of a sharp-edged target (top trace) and the simulated population response profile (bottom trace) with the same parameters as in $C$. The predicted percept (bottom panel) exhibits a slight luminance distortion, but little change in the target position. E, Perceptual extrapolation as a function of the Gaussian width and the target velocity averaged from four subjects (HG, KB, YF, and YD). Plotted was the bilinear interpolation of the data measured at 20 points in the two-dimensional plot, at target widths of $0.5,1,2$, and $4^{\circ}$ and velocities of $1 / 32,1 / 8,1 / 2,4$, and $64^{\circ} / \mathrm{sec}$. We noticed that the magnitude of the perceptual displacement decreases with the number of sessions each subject has performed, which is probably attributable to perceptual learning (Gilbert, 1994; Kapadia et al., 1994). The data shown here were collected in a later phase of the project, which is probably why the magnitude is smaller than that shown in Figure $1 \mathrm{~A}$. $F$, Distance between the centers of mass of the modeled
A

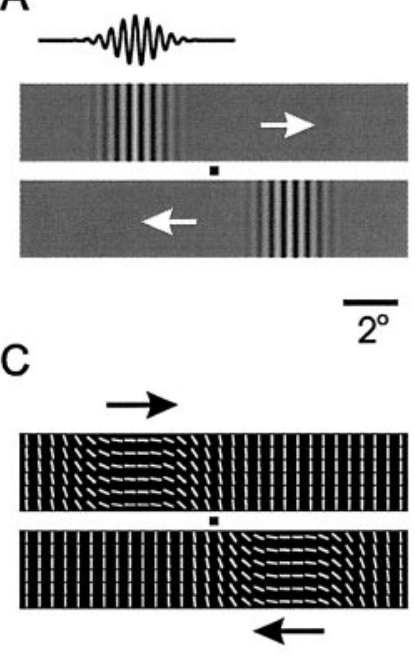

B
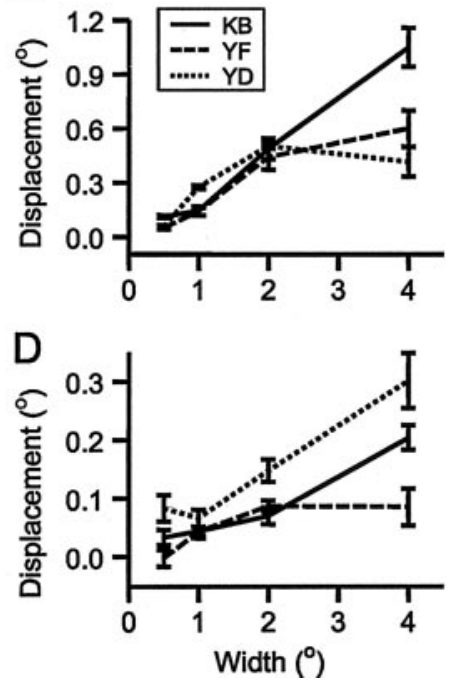

Figure 4. Perceptual extrapolation of second-order moving stimuli. $A, \mathrm{~A}$ second-order target with a Gaussian contrast profile, $L(x, t)=B(1+\exp$ $\left.\left[-(x-v t)^{2} / 2 \sigma^{2}\right] \cos (2 \pi k x)\right)$, where $L$ is the luminance, $B$ is background $\left(50 \mathrm{~cd} / \mathrm{m}^{2}\right), v$ is target velocity, $\sigma$ is the SD of the Gaussian envelope, and $k$ is the spatial frequency of the stationary sinusoidal carrier. $B$, Dependence of the extrapolation on the width of the Gaussian contrast envelope $(W=2.35 \sigma)$, measured at $v=4^{\circ} / \mathrm{sec}$ and $k=2$ cycles $/{ }^{\circ}$. $C, D$, Same as $A$ and $B$, respectively, except the stimuli are line segments for which orientation is modulated by a moving Gaussian profile, $O(x, t)=90^{\circ}-90^{\circ} \exp$ $\left[-(x-v t)^{2} / 2 \sigma^{2}\right]$, where $O$ is the orientation of the line segments (horizontal is defined as $0^{\circ}$ ). The target velocity used for the data in $D$ was $4 \%$ sec. Error bars indicate SEM. Note that the scale of the $y$-axis in $D$ is different from that in $B$ and in Figure $1 D-F$, indicating that the perceptual extrapolation for orientation-defined moving target is qualitatively but not quantitatively similar to that for luminance- or contrast-defined targets.

was a stationary sinusoidal grating at a high spatial frequency, with its contrast modulated by a moving Gaussian envelope (Fig. $4 A$ ). The orientation-defined stimulus consisted of a uniformly distributed array of short line segments, the orientations of which varied according to a moving Gaussian profile (Fig. 4C). With both types of second-order stimuli, subjects perceived a displacement of the targets (Gaussian envelopes) in the direction of motion with the magnitude depending on the Gaussian width, which is a measure of spatial blur for the second-order visual targets (Fig. 4B,D). Thus, blur-dependent perceptual extrapolation occurs for second-order moving targets, which may be mediated by cortical mechanisms (see below).

\section{DISCUSSION}

Perceptual extrapolation of second-order moving targets, in particular the orientation-defined targets, is likely to be mediated by cortical mechanisms. This is because most retinal ganglion cells and thalamic visual neurons in the primate are not orientation selective, and there is no overall luminance difference between the target and the background in these second-order stimuli. The

$\leftarrow$

response profiles for targets moving in opposite directions at the time of target disappearance. Model parameters $\left(b=0.0025 \mathrm{msec}^{-1}, c=\right.$ $0.028, \theta=0.016$ ) were adjusted to fit the data shown in $E$. The plot was generated with the same bilinear interpolation method, from simulated data at the same set of velocities and target widths as in E. White broken line, optimal velocity for perceptual displacement as a function of target width. Color bar indicates the magnitude of the perceptual displacement. 
biphasic temporal response property, which allows a visual neuron to compute the temporal derivative of the "effectiveness" of the stimulus, is widely observed along the visual pathway (Shapley and Victor, 1978; Saul and Humphrey, 1990), including in the visual cortex (DeAngelis et al., 1993; Ringach et al., 1997). For the orientation-defined stimulus shown in Figure $4 C$, the target position can be represented by the population response profile of cortical neurons selective for the horizontal orientation. With biphasic temporal responses to oriented stimuli (Ringach et al., 1997), these neurons should exhibit higher responses to the leading side of the moving target as the local orientation turns toward horizontal (becoming more effective) and lower responses to the trailing side of the target as the orientation turns away from horizontal. This may result in a shift of the cortical response profile in the direction of target motion and hence perceptual extrapolation of the target position (Fig. $3 C$ ).

We have shown that the motion-induced perceptual extrapolation of both first- and second-order targets depends critically on spatial blurring of the targets (Figs. 1, 4B,D). Both the blur dependence and the relatively low optimal target velocity of the effect (Figs. 2A, 3E) indicate that this form of perceptual extrapolation is distinct from the motion-extrapolation mechanism originally proposed to account for the flash-lag illusion (Nijhawan, 1994). These properties can also explain why previous studies using similar motion-stop paradigms did not reveal significant perceptual extrapolation (Eagleman and Sejnowski, 2000; Krekelberg and Lappe, 2000), because their visual targets had sharp edges and higher velocities. In the model shown in Figure 3, the blur dependence of the perceptual extrapolation has a simple explanation: the biphasic temporal response property of individual visual neurons causes a distortion of the spatial profile of the population neuronal response to moving targets. This distortion results in a shift in the overall position of the response profile in the direction of motion for blurred targets (Fig. 3C), but not for sharp-edged targets (Fig. 3D). In the rabbit and salamander retina, shift in the neuronal response profile in the direction of target motion has been shown for sharp-edged targets (Berry et al., 1999). This is attributable to the relatively large receptive fields of the retinal ganglion cells used in that study, which effectively blurred the visual stimuli. Because the human visual system has a higher spatial resolution and the cells have smaller receptive fields, actual blurring of the visual targets becomes a critical requirement for the perceptual extrapolation.

Blurred edges are common in natural scenes; imperfect focus and constant tremor of the eye can introduce further blur when signals reach the retina. Previous studies have characterized perceptual localization of stationary blurred edges in human vision (Watt and Morgan, 1983; Morgan et al., 1984). Here, we have demonstrated a perceptual mislocalization of blurred stimulus induced by motion. Interaction between motion and perceptual localization has been demonstrated in several forms (Ramachandran and Anstis, 1990; De Valois and De Valois, 1991; Snowden, 1998; Nishida and Johnston, 1999; Whitney and Cavanagh, 2000), most of which are thought to be mediated by recurrent connections between motion-processing visual areas (e.g., medial temporal cortex) and the primary visual cortex. Here, the blurdependent spatial extrapolation of moving stimuli represents another form of motion-position interaction, which is presumably caused by a simple temporal response property of visual neurons. Given the ubiquity of the biphasic temporal response mechanism in neuronal circuits, this form of perceptual extrapolation may be supported at various levels and modalities of sensory processing.

\section{REFERENCES}

Baldo MV, Klein SA (1995) Extrapolation or attention shift? Nature 378:565-566.

Berry II MJ, Brivanlou IH, Jordan TA, Meister M (1999) Anticipation of moving stimuli by the retina. Nature 398:334-338.

Brenner E, Smeets JB (2000) Motion extrapolation is not responsible for the flash-lag effect. Vision Res 40:1645-1648.

Cavanagh P, Mather G (1989) Motion: the long and short of it. Spat Vis 4:103-129.

DeAngelis GC, Ohzawa I, Freeman RD (1993) Spatiotemporal organization of simple-cell receptive fields in the cat's striate cortex. I General characteristics and postnatal development. J Neurophysiol 69:1091-1117.

De Valois RL, De Valois KK (1991) Vernier acuity with stationary moving Gabors. Vision Res 31:1619-1626.

Eagleman DM, Sejnowski TJ (2000) Motion integration and postdiction in visual awareness. Science 287:2036-2038.

Gescheider GA (1997) Psychophysics: the fundamentals. Mahwah, NJ: Lawrence Erlbaum.

Gilbert CD (1994) Early perceptual learning. Proc Natl Acad Sci USA 91:1195-1197.

Kapadia MK, Gilbert CD, Westheimer G (1994) A quantitative measure for short-term cortical plasticity in human vision. J Neurosci 14:451-457.

Krekelberg B, Lappe M (1999) Temporal recruitment along the trajectory of moving objects and the perception of position. Vision Res 39:2669-2679.

Krekelberg B, Lappe M (2000) A model of the perceived relative positions of moving objects based upon a slow averaging process. Vision Res 40:201-215.

Lappe M, Krekelberg B (1998) The position of moving objects. Perception 27:1437-1449.

MacKay DM (1958) Perceptual stability of a stroboscopically lit visual field containing self-luminous objects. Nature 181:507-508.

Morgan MJ, Mather G, Moulden B, Watt RJ (1984) Intensity-response nonlinearities and the theory of edge localization. Vision Res 24:713-719.

Nijhawan R (1994) Motion extrapolation in catching. Nature 370:256-257.

Nijhawan R (1997) Visual decomposition of colour through motion extrapolation. Nature 386:66-69.

Nishida S, Johnston A (1999) Influence of motion signals on the perceived position of spatial pattern. Nature 397:610-612.

Purushothaman G, Patel SS, Bedell HE, Ogmen H (1998) Moving ahead through differential visual latency. Nature 396:424.

Ramachandran VS, Anstis SM (1990) Illusory displacement of equiluminous kinetic edges. Perception 19:611-616.

Ringach DL, Hawken MJ, Shapley R (1997) Dynamics of orientation tuning in macaque primary visual cortex. Nature 387:281-284.

Saul AB, Humphrey AL (1990) Spatial and temporal response properties of lagged and nonlagged cells in cat lateral geniculate nucleus. J Neurophysiol 64:206-224.

Shapley RM, Victor JD (1978) The effect of contrast on the transfer properties of cat retinal ganglion cells. J Physiol (Lond) 285:275-298.

Snowden RJ (1998) Shifts in perceived position following adaptation to visual motion. Curr Biol 8:1343-1345.

Somers DC, Todorov EV, Siapas AG, Toth LJ, Kim DS, Sur M (1998) A local circuit approach to understanding integration of long-range inputs in primary visual cortex. Cereb Cortex 8:204-217.

Watt RJ, Morgan MJ (1983) The recognition and representation of edge blur: evidence for spatial primitives in human vision. Vision Res 23:1465-1477.

Whitaker D, Pearson S, McGraw PV, Banford M (1998) Keeping a step ahead of moving objects. IOVS [Suppl] 39:S1078.

Whitney D, Cavanagh P (2000) Motion distorts visual space: shifting the perceived position of remote stationary objects. Nat Neurosci 3:954-959.

Whitney D, Murakami I (1998) Latency difference, not spatial extrapolation. Nat Neurosci 1:656-657.

Whitney D, Murakami I, Cavanagh P (2000) Illusory spatial offset of a flash relative to a moving stimulus is caused by differential latencies for moving and flashed stimuli. Vision Res 40:137-149. 